\title{
Evaluation of the Acromioclavicular Joint Morphology for Minimizing Subacromial Erosion after Surgical Fixation of the Joint Using a Clavicular Hook Plate
}

\author{
Sung-Jae Kim ${ }^{1}$, Young-Moon Kee ${ }^{2}$, Dong-Hyuck Park, Young-Il Ko, Bong-Gun Lee \\ Department of Orthopaedic Surgery, Hanyang University College of Medicine, Seoul, ${ }^{1}$ Department of Orthopaedic Surgery, Dongtan Sacred Heart Hospital, Hallym \\ University College of Medicine, Hwaseong, ${ }^{2}$ Department of Orthopaedic Surgery, Gwangmyeong Saeum Hospital, Gwangmyeong, Korea
}

\begin{abstract}
Background: Subacromial erosion remains a major concern after surgical fixation of acromioclavicular (AC) joint using a clavicular hook plate. To minimize postoperative subacromial erosion, we investigated the structural relationship between distal clavicle and acromion around the AC joint by considering the surgical fixation of the joint using the hook plate technique.

Methods: Computed tomography scans of 101 AC joints without any inherent pathology were analyzed. The angle between the distal clavicle and acromion around the AC joint (AC angle), depth of the acromion, differences in height between distal clavicle and acromion (AC height difference), and thickness of distal clavicle and acromion at the AC joint were measured. Descriptive statistics were calculated for each anatomical parameter, and all results were compared between gender groups.

Results: The mean $\mathrm{AC}$ angle was $17.1^{\circ}$ (range, $-8.0^{\circ}$ to $39.0^{\circ}$ ), and the mean AC height difference was $3.5 \mathrm{~mm}$ (range, -0.7 to $8.7 \mathrm{~mm}$ ). Both factors showed very high variability (coefficients of variation $=62.6 \%$ and $46.6 \%$, respectively). The mean AC angle was significantly higher in the female gender than in the male gender $\left(19.8^{\circ}\right.$ vs. $\left.13.8^{\circ}, p=0.048\right)$. The mean acromion thickness and distal clavicular thickness were both significantly thinner in the female group than in the male group $(p<0.001)$.

Conclusions: Taken together, we believe our results might be helpful in minimizing postoperative subacromial erosion when performing surgical fixation of the AC joint using the hook plate, and be valuable in improving future design of the hook plate.
\end{abstract}

(Clin Shoulder Elbow 2018;21(3):138-144)

Key Words: Acromioclavicular joint; Joint dislocations; Shoulder fractures; Hook plate; Subacromial erosion

\section{Introduction}

Surgical fixation of the acromioclavicular (AC) joint dislocation or unstable fracture of the distal clavicle involves a complicated procedure for orthopedic surgeons. The recently developed clavicular hook plate is designed to be fixed on the distal clavicle with its hook being inserted beneath the acromion, just posterior to the AC joint. The hook works as a lever for depressing the clavicle to the level of the acromion at the AC joint, and facilitate bony or ligamentous healing. ${ }^{1,2)}$ Several studies have described favorable surgical outcomes and fast bony union or ligamentous healing with few complications. ${ }^{2-7)}$ However, complications such as postoperative pain, acromial erosion (Fig. 1), or fractures are known to occur. ${ }^{8-12)}$ To minimize these complications, the pressure between the hook tip and undersurface of the acromion needs to be dispersed. ${ }^{2,8)}$ Since the designing of the hook plate requires it to be placed across the distal clavicle and the acromion around the AC joint, a thorough knowledge of the structural relationship between these two bony structures is important for the safe positioning. Although one previous study described the surgical anatomy around the AC joint in relation to the hook plate implantation, ${ }^{2)}$ it mainly focused on the anatomy of the

Received June 7, 2018. Revised July 6, 2018. Accepted July 8, 2018.

Correspondence to: Bong-Gun Lee

Department of Orthopaedic Surgery, Hanyang University College of Medicine, 222 Wangsimni-ro, Seongdong-gu, Seoul 04763, Korea

Tel: +82-2-2290-8485, Fax: +82-2-2299-3774, E-mail: Ibgwej@hanmail.net, ORCID: https://orcid.org/0000-0002-4003-5529

IRB approval: Hanyang University Hospital (HYUH 2018-05-029-001).

Financial support: Bong-Gun Lee has received a grant (HY-2012-N) from Hanyang University. Conflict of interests: None. 


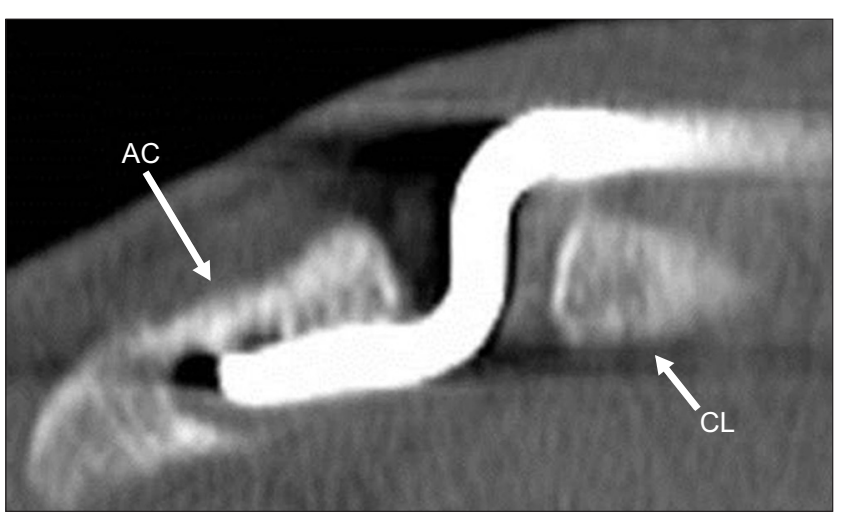

Fig. 1. Radiograph taken at 2 months following surgery shows marked erosion of undersurface of the AC.

AC: acromioclavicular, CL: clavicle.

horizontal plane of the acromion and did not include the coronal plane structural relationship between these two bones. The current study therefore aims to investigate the in vivo coronal plane structural relationship between the distal clavicle and acromion around the AC joint using computed tomography (CT) of the shoulder joint. We believe that the results generated from the data would help in the selection and bending of the hook plate, and be valuable to facilitate in improving the future design of the hook plate from the perspective of surgical fixation of the $\mathrm{AC}$ joint using this technique.

\section{Methods}

\section{Patient Selection}

Between January 2007 and October 2011, 427 patients who underwent $\mathrm{CT}$ evaluations for $\mathrm{AC}$ joint at our institute were eligible for the current study. The inclusion criteria enrolled patients with CT scans comprising clear images of the AC joint without any pathology. Exclusion criteria were as follows: AC joint arthritis, glenohumeral joint arthritis, fracture in the scapula or the clavicle, previous history of AC joint injury, and presence of os acromiale. The CT images inappropriate for analysis were also excluded. Of the 427 cases considered, 101 cases were included in the final analysis. Diagnosis of the patients enrolled in the present study are listed in Table 1. CT scans were done for the patients with anterior instability to assess the bony defect on the glenoid. There were 61 male and 40 female patients; 72 were right side and 29 were left side cases. Mean age of patients was 25.8 years (range, 16 to 39 years; standard deviation [SD], $5.88)$.

\section{Computed Tomography Scanning}

CT scan was performed with a spiral, double-slice CT system (Brilliance 64; Philips Electronics, Eindhoven, Netherlands). Using a Y-shaped filter, $1 \mathrm{~mm}$ slices were produced with a slice
Table 1. Diagnoses of the Patients

\begin{tabular}{lc}
\hline \multicolumn{1}{c}{ Diagnosis } & No. of case \\
\hline Anterior instability of the shoulder joint & $57(56.4)$ \\
Bony neoplasm of the proximal humerus of the scapula & $19(18.8)$ \\
Shoulder contusion without fracture & $10(9.9)$ \\
AC joint appeared on chest CT images & $15(14.9)$ \\
Total & $101(100)$ \\
\hline
\end{tabular}

Values are presented as number (\%).

AC: acromioclavicular, CT: computed tomography.

Table 2. Anatomical Parameters Used in the Present Study

\begin{tabular}{ll}
\hline \multicolumn{1}{c}{ Parameters } & \multicolumn{1}{c}{ Definition } \\
\hline $\begin{array}{l}\text { Acromioclavicular } \\
\text { (AC) angle }\end{array}$ & $\begin{array}{l}\text { The angle between the upper surface of the distal } \\
\text { clavicle at the AC joint and lower surface of the } \\
\text { acromion just posterior to the AC joint (Fig. 2A-C) }\end{array}$ \\
$\begin{array}{l}\text { Depth of the } \\
\text { acromion }\end{array}$ & $\begin{array}{l}\text { The vertical distance between the upper margin of the } \\
\text { distal clavicle at the AC joint and lower margin of the } \\
\text { acromion just posterior to the AC joint (Fig. 2D) }\end{array}$ \\
$\begin{array}{l}\text { AC height } \\
\text { difference }\end{array}$ & $\begin{array}{l}\text { The distance between the upper margin of the distal } \\
\text { clavicle and upper margin of the acromion at the AC } \\
\text { joint (Fig. 3) }\end{array}$ \\
\hline
\end{tabular}

${ }^{\star}$ Acute angle was used for measuring the AC angle.

${ }^{\dagger}$ Positive value means the distal clavicle is higher than the acromion.

increment of $0.5 \mathrm{~mm}$, at $350 \mathrm{~mA}$ and $140 \mathrm{kV}$, with a matrix of $512 \times 512$ pixels. All images were obtained with the patient in the supine position.

\section{Measurement of the Anatomical Parameters}

Anatomical parameters used in present study were measured in the coronal plane of the CT images and are summarized in Table 2. The measurement protocol of the anatomical parameters is described in Fig. 2 and 3. We also assessed the thicknesses of the acromion and the distal clavicle. All the measurements were performed using the PiviewSTAR measurement tools ( $\mathrm{Pi}$ view 5.08; Infinitt, Seoul, Korea). For assessing the reliability, all measurements were continually done by a single observer, and repeated by another independent observer blinded to the prior observer's results. Descriptive statistics were performed for each anatomical parameter. All results were then compared between the gender groups.

\section{Statistical Analysis}

Intraclass correlation coefficients for continuous variables and Cohen's kappa for nominal variables were used for estimating the inter- and intra-observer reliability. Interpretation of the Kappa value was based on the criteria provided by Landis and Koch $^{13)}: \geq 0.81$, almost perfect agreement; 0.61 to 0.80 , substantial; 0.41 to 0.60 , moderate; 0.21 to 0.40 , fair; 0 to 0.20 , slight. The following criteria were used for interpreting intraclass 

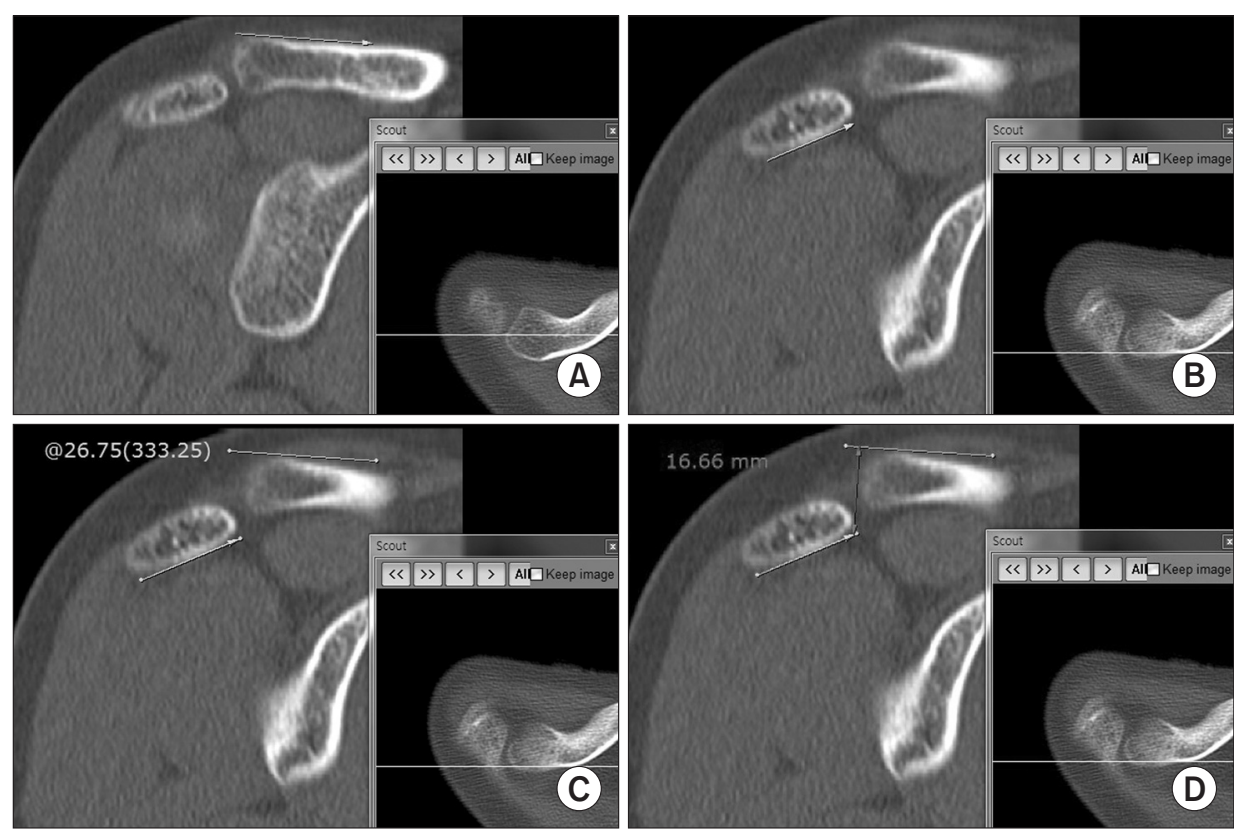

Fig. 2. To measure the acromioclavicular (AC) angle, first draw a reference line on the upper surface of the distal clavicle on the coronal plane image (A); the scouter line on the axial plane image appears on the belowright corner and is located at the center of the AC joint. Next, draw another reference line on the lower surface of the acromion just posterior to the $\mathrm{AC}$ joint (B); the scouter line on the axial plane image is located just posterior to the AC joint. The measurement tool allows to measure the angle between the two reference lines, $26.75^{\circ}$ in this case (C). To measure the depth of the acromion, the line perpendicular to the reference line on the distal clavicle is drawn just lateral to the distal clavicle (D), then measure the distance between two intersecting points.

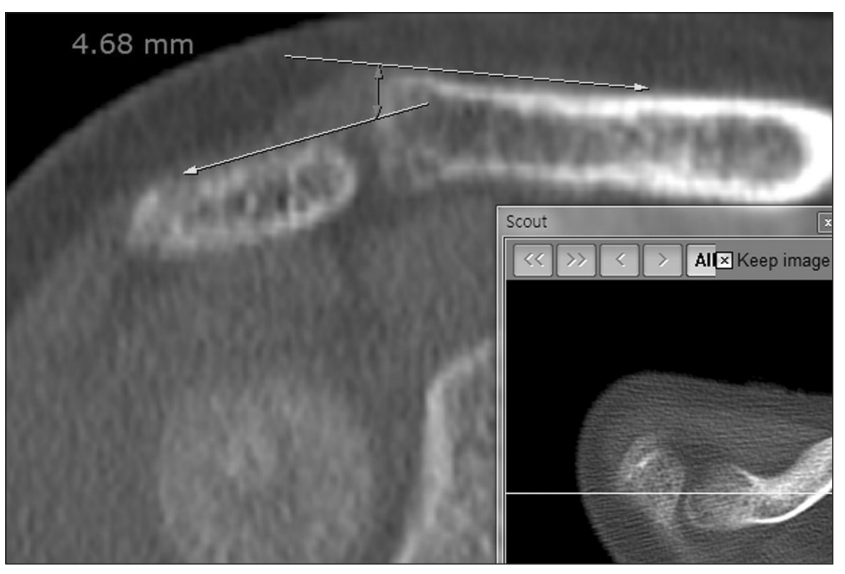

Fig. 3. To measure the acromioclavicular (AC) height difference, draw two reference lines on the upper surface of the distal clavicle and the acromion; the scouter line on the axial plane image is located at the center of the AC joint, then draw another line perpendicular to the reference line on the distal clavicle, and measure the distance between to intersection points.

correlation coefficient values: 1 , perfect reliability; 0.8 to 1 , very good; 0.61 to 0.8 , good; 0.41 to 0.6 , moderate; $\leq 0.4$, poor. ${ }^{14)}$ To analyze the individual variability of the anatomical parameters, we estimated coefficients of variation (CV) for each parameter: $<10 \%$ low variation, $10 \%$ to $20 \%$ medium variation, $20 \%$ to $30 \%$ high variation, and $>30 \%$ very high variation. ${ }^{15)}$ The independent two sample Student t-test compared the continuous variables, and the Mann-Whitney U-test compared the ordinal variables between the gender groups. The chi-square test and Fisher exact test were used to compare variables with nominal scales. The SPSS software package ver. 16.0 (SPSS Inc., Chicago, IL, USA) was used for statistical analyses. The level of significance was set at 0.05 .

\section{Results}

\section{Measurement Reliability}

The intra-observer reliability of repeated measurements ranged from 0.816 to 0.945 , and the inter-observer reliability ranged from 0.702 to 0.926 . Our results demonstrate that all measurements done in the current study have good to excellent measurement reliability. ${ }^{13,14)}$

\section{Anatomical Parameters}

The anatomical parameters evaluated are listed in Table 3. The mean $\mathrm{AC}$ angle was $17.1^{\circ} \pm 10.51^{\circ}$, ranging from $-8^{\circ}$ to $39^{\circ}$ with very high variability $(C V=62.6 \%)$. The mean $A C$ height difference was $3.5 \pm 1.65 \mathrm{~mm}$ and ranged between -0.7 to 8.7 with a very high variability ( $\mathrm{CV}=46.6 \%)$. Of the 101 cases evaluated, 100 cases (99.0\%) showed positive AC height difference, and 1 case showed negative AC height difference $(-0.7 \mathrm{~mm})$. The depth of the acromion, thickness of the acromion, and the distal clavicle all showed medium variability $(C V=16.6 \%$, $15.0 \%$, and $12.9 \%$, respectively).

\section{Differences of the Anatomical Parameters between Genders}

Comparison of anatomical parameters between genders are listed in Table 4. The mean AC angle is significantly higher in female than in male gender $(p=0.048)$. The mean depth of the acromion and mean AC height difference were significantly greater in male than in female gender $(p=0.001$ and 0.013 , respectively). The mean acromion thickness and the distal clavicular thickness were significantly thinner in the female group than male group ( $p<0.001$ for both factors). 
Table 3. Anatomical Parameters in Normal Population

\begin{tabular}{|c|c|c|c|}
\hline Variable & Mean \pm SD (range) & $95 \% \mathrm{CI}$ & $\mathrm{CV}^{*}(\%)$ \\
\hline AC angle $\left({ }^{\circ}\right)$ & $17.1 \pm 10.51(-8.0$ to 39.0$)$ & 14.99 to 19.17 & 62.6 \\
\hline Depth of the acromion (mm) & $10.9 \pm 1.80(5.5$ to 15.6$)$ & 10.51 to 11.22 & 16.6 \\
\hline AC height difference (mm) & $3.5 \pm 1.65(-0.7$ to 8.7$)$ & 3.22 to 3.88 & 46.6 \\
\hline Acromion thickness (mm) & $8.3 \pm 1.07(5.7$ to 11.4$)$ & 8.10 to 8.53 & 12.9 \\
\hline Distal clavicle thickness (mm) & $11.7 \pm 1.76(8.5$ to 16.7$)$ & 11.33 to 12.03 & 15.0 \\
\hline
\end{tabular}

SD: standard deviation, CI: confidence interval, CV: coefficients of variation, AC: acromioclavicular.

${ }^{*} \mathrm{CV}$ below $10 \%$ : low variation, $10 \%-20 \%$ : medium variation, $20 \%-30 \%$ : high variation, above $30 \%$ : very high variation.

Table 4. Comparison of Anatomical Parameters between Gender Groups

\begin{tabular}{lccc}
\hline \multicolumn{1}{c}{ Variable } & Male $(\mathrm{n}=61)$ & Female $(\mathrm{n}=40)$ & $p$-value \\
\hline Mean age $(\mathrm{yr})$ & $25.2 \pm 5.50(18$ to 38$)$ & $27.6 \pm 6.48(18$ to 39$)$ & $0.058^{\dagger}$ \\
\hline AC angle $\left(^{\circ}\right)$ & $13.8 \pm 10.09(-4$ to 35$)$ & $19.8 \pm 10.73(-8$ to 45$)$ & $0.048^{*, \dagger}$ \\
\hline Depth of the acromion $(\mathrm{mm})$ & $11.3 \pm 1.77(7.9$ to 15.6$)$ & $10.2 \pm 1.64(5.5$ to 12.1$)$ & $0.001^{*, \dagger}$ \\
\hline AC height difference $(\mathrm{mm})$ & $3.9 \pm 1.65(1.4$ to 8.6$)$ & $10.9 \pm 1.37(8.5$ to 14.3$)$ & $0.013^{*, *}$ \\
\hline Distal clavicle thickness $(\mathrm{mm})$ & $12.2 \pm 1.81(9.0$ to 16.6$)$ & $7.7 \pm 0.94(5.7$ to 9.8$)$ & $<0.001^{*, *}$ \\
\hline Acromion thickness $(\mathrm{mm})$ & $8.7 \pm 0.95(7.3$ to 11.3$)$ & $<0.001^{\text {*,* }}$ \\
\hline
\end{tabular}

Values are presented as mean \pm standard deviation (range).

AC: acromioclavicular.

${ }^{*} p<0.05$.

${ }^{\dagger}$ Mann-Whitney U-test.

${ }^{\ddagger}$ Student t-test.

\section{Retrospective Power Analysis}

We performed a retrospective power analysis considering the $\mathrm{AC}$ angle as the primary outcome measurement. With the statistical model of two-tailed independent two sampled Student t-test, the standardized effect size of this statistical analysis is $0.576(19.8-13.8 / 10.42 ; 10.42$ is a pooled SD of the two gender groups). With the significance level at 0.05 , the power of this statistical analysis was calculated as $80.1 \%$.

\section{Discussion}

The current study analyzed the coronal plane structural anatomy around the AC joint and its variability between individuals, and evaluated the differences between genders. Furthermore, we propose several clinically relevant anatomical parameters in the perspective of surgical fixation of the AC joint using the hook plate.

Although several authors account complications related to the stress concentration on the undersurface of the acromion after hook plate fixation, ${ }^{8,9,16)}$ surgical anatomy of the AC joint in relation to the hook plate fixation is seldom reported in literature, except one previous study. ElMaraghy et al. ${ }^{2)}$ analyzed the acromion anatomy of 15 cadaveric samples using a threedimensional model. They analyzed the antero-posterior width at the lateral aspect of the acromion, medial to lateral length of the acromion, and its thickness. They concluded that these anatomical parameters are unique for each patient, and therefore 'standard' acromial dimensions could not be found. Another conclusion was that a substantial portion of the samples revealed the hook to be in contact with the supraspinatus tendon, subacromial bursa, and acromial bone, with stress concentrated at the hook tip. This study is relevant since it evidences the concerns of subacromial structural damage after hook plate fixation. However, the study did not include the clinically relevant coronal plane structural relationship between the distal clavice and the acromion during execution of the hook plate technique.

In the current study, the angle between the distal clavicle at the $\mathrm{AC}$ joint and the acromion just posterior to the $\mathrm{AC}$ joint was observed to be variable (AC angle, $16.8^{\circ} \pm 10.38^{\circ}$ ). Since the plate to be fixed on the distal clavicle and the hook to be inserted beneath the acromion are designed as a fixed angle in shape, the point of contact between the hook tip and the undersurface of the acromion seems inevitable in many cases (Fig. 4). The hook plate is designed such that it maintains the normal biomechanics of the AC joint, and the hook portion is free to move during arm elevation. ${ }^{17)}$ Many authors suggest that if the point contact between hook tip and undersurface of the acromion occurs, this movement of the hook under the acromion might con- 
sequently result in bony erosion. ${ }^{2,8,10,18,19)}$ Hence, to avoid stress concentration between the hook tip and the undersurface of the acromion, bending of the hook portion or plate portion of the plate in accordance with the AC angle might be necessitated. We therefore suggest that the AC angle, which shows very high individual variability in our results, should be thoroughly examined for each patient (Fig. 2, 5).

The depth of the acromion at the AC joint might be important when choosing the depth of the acromial hook plate. Inserting a plate with smaller depth of the hook portion than the depth of the acromion could result in over-reduction of the AC joint as well as occurrence of pin point contact between the hook tip and the acromion. Many studies recommend using an accurate depth of the hook of the plate to avoid excessive pressure on the undersurface of the acromion. ${ }^{3,8,18)}$

In terms of AC height difference, the mean value in the current study was $3.5 \mathrm{~mm}$, and almost all cases showed positive values (except 1 case with $-0.7 \mathrm{~mm}$ ). This indicates that the distal clavicle is usually located at a higher position than the acromion in the AC joint. It might be clinically important for determining the proper reduction of the $\mathrm{AC}$ joint. For example, in $\mathrm{AC}$ joints

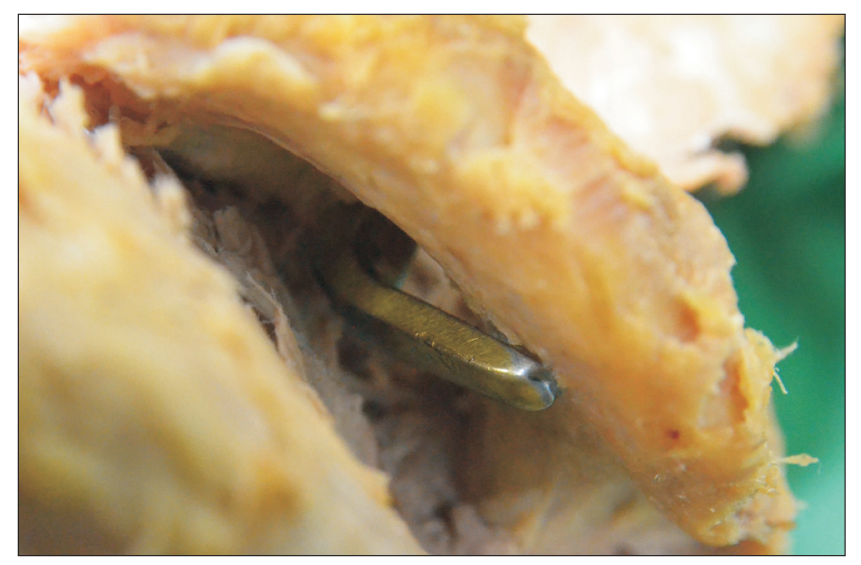

Fig. 4. Cadaveric demonstration of the lateral aspect of the acromion with the deltoid muscle completely detached; the pin point contact between the hook tip and the under surface of the acromion is obvious. with large positive AC height difference before injury, neutralization of the $\mathrm{AC}$ height difference after surgical fixation could indicate excessive depression of the distal clavicle. Over-reduction of the AC joint causes excessive stress on the distal clavicle, and especially on the point contact area of the acromion. The large $\mathrm{AC}$ angle, inappropriate selection of the depth of the hook, and over-reduction of the $\mathrm{AC}$ joint result from the inconsequential to the normal positive $\mathrm{AC}$ height difference, and all these factors aggravate the stresses imposed on the undersurface of the acromion by the hook.

We observed significant differences between genders in the anatomy around the AC joint, which we believe to be a clinically relevant observation. Similarly, Wisanuyotin et al. ${ }^{20)}$ also reported that the anatomy of the clavicle is significantly different between genders. In the present study, the female gender has a significantly larger AC angle $\left(13.8^{\circ}\right.$ vs. $\left.19.8^{\circ}\right)$, and thinner distal clavicle (12.2 mm vs. $10.9 \mathrm{~mm}$ ) and acromion (8.7 mm vs. 7.7 $\mathrm{mm}$ ) as compared to the male gender. This might indicate that the female patients are at a higher risk of having complications related to the stresses on the undersurface of the acromion, although we do not have evidences to prove this assumption. ElMaraghy et al. ${ }^{2)}$ similarly suggests that females might have more complications after surgical fixation of the AC joint using the hook plate. They describe that despite the hook being positioned posterior to the AC joint, the base of the hook still rests in the middle of supraspinatus fossa, which may subsequently lead to subacromial impingement between the hook and the supraspinatus tendon. Further studies analyzing the relationship between the occurrence of postoperative complications and the degree of the $\mathrm{AC}$ angle or the gender are required to validate this hypothesis.

The current study has many limitations. The main drawback of this study is that we could not conduct a statistical comparison between the groups with and without subacromial erosion after surgical fixation with the hook plate. After experiencing the first case with subacromial erosion subsequent to the use of the hook plate, we initiated bending the hook of the plate prior to final fixation in accordance with the AC angle assessed with in-
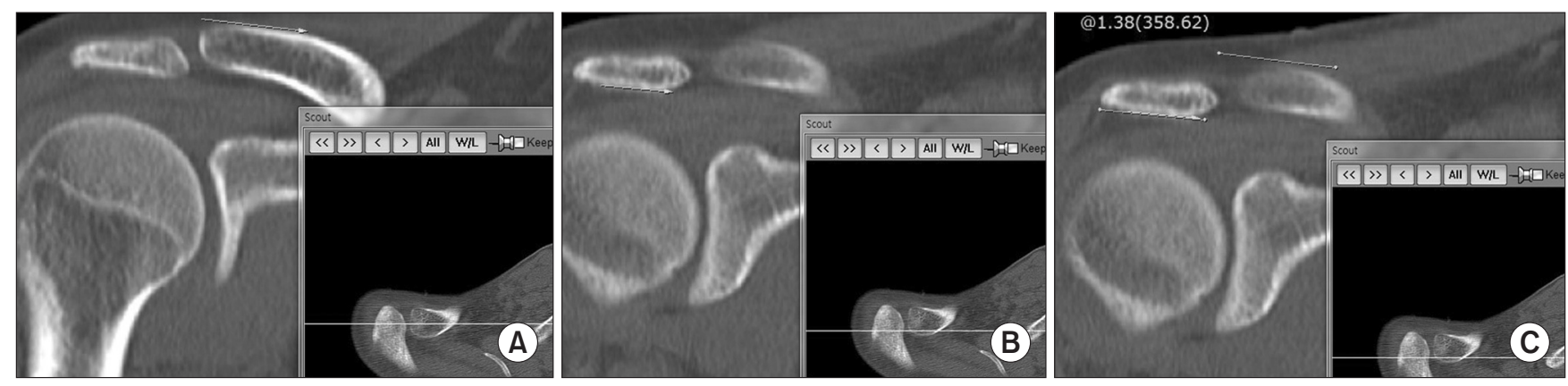

Fig. 5. Measurement of the acromioclavicular (AC) angle in another case. Draw the first reference line in the upper surface of the distal clavicle at the AC joint (A), the second reference line on the lower surface of the acromion at just posterior to the $\mathrm{AC}$ joint (B); the angle between two lines is almost parallel (1.38 $\left.{ }^{\circ}\right)$ in this case $(\mathrm{C})$. 
traoperative fluoroscope. Hence, there are insufficient cases with subacromial erosion for performing the statistical comparison to confirm that large AC angle is a prognostic factor for subacromial erosion. Second, since there are no prior literatures describing the $\mathrm{AC}$ angle that can be used as a reference value for sample size calculation in comparison between gender groups, we were unable to perform sample size calculation before collecting data. However, we performed a retrospective power analysis for the AC angle between the two gender groups. The result showed the beta error probability of the analysis was low enough for a clinical study (power $=80.1 \%$ ). Third, our measurements to estimate the structural relationship between the acromion and the distal clavicle were based only on the coronal plane, which may not be the correct representation of the three-dimensional relationship. Fourth, all the CT scans were performed on patients in the supine position; thus, our findings may differ slightly from scans performed in the standing or sitting position. Fifth, this study includes the shoulder joints with anterior instability, contusion, and neoplasm, which may cause subtle abnormalities in the structural relationship at the AC joint. However, we used strict criteria in selecting subjects in order to reflect the closest to normal anatomy of the AC joint. In spite of all these limitations, we believe that our observations could be helpful in improving the future design of the hook plate, and also be the corner stone for future studies investigating the relationship between the postoperative acromial complications and the anatomy around the AC joint.

\section{Conclusion}

The angle between the distal clavicle and the acromion around the AC joint is not parallel in many cases, and there are high individual variabilities in this angle. The distal clavicle seems to be usually located higher than the acromion at the AC joint. The female gender has a greater angle between the distal clavicle and the acromion, and thinner acromion and distal clavicle around the AC joint. During surgical fixation of the AC joint using the hook plate, considering these anatomies may help in selecting and bending the hook plate. Moreover, our results may help improve the future designs of the hook plate for minimizing postoperative acromial erosion.

\section{References}

1. Lin HY, Wong PK, Ho WP, Chuang TY, Liao YS, Wong CC. Clavicular hook plate may induce subacromial shoulder impingement and rotator cuff lesion: dynamic sonographic evaluation. J Orthop Surg Res. 2014;9:6.

2. EIMaraghy AW, Devereaux MW, Ravichandiran K, Agur AM. Subacromial morphometric assessment of the clavicle hook plate. Injury. 2010;41(6):613-9.
3. Muramatsu K, Shigetomi M, Matsunaga T, Murata Y, Taguchi T. Use of the AO hook-plate for treatment of unstable fractures of the distal clavicle. Arch Orthop Trauma Surg. 2007;127(3): 191-4.

4. Meda PV, Machani B, Sinopidis C, Braithwaite I, Brownson P, Frostick SP. Clavicular hook plate for lateral end fractures: a prospective study. Injury. 2006;37(3):277-83.

5. Flinkkilä T, Ristiniemi J, Hyvönen $P$, Hämäläinen M. Surgical treatment of unstable fractures of the distal clavicle: a comparative study of Kirschner wire and clavicular hook plate fixation. Acta Orthop Scand. 2002;73(1):50-3.

6. Faraj AA, Ketzer B. The use of a hook-plate in the management of acromioclavicular injuries. Report of ten cases. Acta Orthop Belg. 2001;67(5):448-51.

7. Tiren D, van Bemmel AJ, Swank DJ, van der Linden FM. Hook plate fixation of acute displaced lateral clavicle fractures: midterm results and a brief literature overview. J Orthop Surg Res. $2012 ; 7: 2$.

8. Chiang CL, Yang SW, Tsai MY, Kuen-Huang Chen C. Acromion osteolysis and fracture after hook plate fixation for acromioclavicular joint dislocation: a case report. J Shoulder Elbow Surg. 2010;19(4):e13-5.

9. Nadarajah R, Mahaluxmivala J, Amin A, Goodier DW. Clavicular hook-plate: complications of retaining the implant. Injury. 2005;36(5):681-3.

10. Kim YS, Yoo YS, Jang SW, Nair AV, Jin H, Song HS. In vivo analysis of acromioclavicular joint motion after hook plate fixation using three-dimensional computed tomography. J Shoulder Elbow Surg. 2015;24(7):1106-11.

11. Zhang C, Huang J, Luo Y, Sun H. Comparison of the efficacy of a distal clavicular locking plate versus a clavicular hook plate in the treatment of unstable distal clavicle fractures and a systematic literature review. Int Orthop. 2014;38(7):1461-8.

12. Gu X, Cheng B, Sun J, Tao K. Arthroscopic evaluation for omalgia patients undergoing the clavicular hook plate fixation of distal clavicle fractures. J Orthop Surg Res. 2014;9:46.

13. Landis JR, Koch GG. The measurement of observer agreement for categorical data. Biometrics. 1977;33(1):159-74.

14. Hirschmann MT, Konala P, Amsler F, Iranpour F, Friederich NF, Cobb JP. The position and orientation of total knee replacement components: a comparison of conventional radiographs, transverse 2D-CT slices and 3D-CT reconstruction. J Bone Joint Surg Br. 2011;93(5):629-33.

15. Couto MF, Peternelli LA, Barbosa MHP. Classification of the coefficients of variation for sugarcane crops. Ciência Rural. 2013; 43(6):957-61.

16. Chen $C Y$, Yang SW, Lin $K Y$, et al. Comparison of single coracoclavicular suture fixation and hook plate for the treatment of acute unstable distal clavicle fractures. J Orthop Surg Res. 2014;9:42.

17. Fung $M$, Kato S, Barrance PJ, et al. Scapular and clavicular 
kinematics during humeral elevation: a study with cadavers. J Shoulder Elbow Surg. 2001;10(3):278-85.

18. Hoffler CE, Karas SG. Transacromial erosion of a locked subacromial hook plate: case report and review of literature. J Shoulder Elbow Surg. 2010;19(3):e12-5.
19. Chaudry SN, Waseem M. Clavicular hook plate: complications of retaining the implant. Injury. 2006;37(7):665.

20. Wisanuyotin T, Tidchom C, Chaisiwamonkhol K, et al. Geometry of the clavicle and reliability of measurement using PACS. Surg Radiol Anat. 2014;36(6):573-7. 